\title{
The Imprint of Dissipation on the Shapes of Merger Remnant Losvds
}

\section{Citation}

Hoffman, Loren, Thomas J. Cox, Suvendra Dutta, and Lars Hernquist. 2009. "THE IMPRINT OF DISSIPATION ON THE SHAPES OF MERGER REMNANT LOSVDs." The Astrophysical Journal 705 (1): 920-25. https://doi.org/10.1088/0004-637x/705/1/920.

\section{Permanent link}

http://nrs.harvard.edu/urn-3:HUL.InstRepos:41381851

\section{Terms of Use}

This article was downloaded from Harvard University's DASH repository, and is made available under the terms and conditions applicable to Open Access Policy Articles, as set forth at http:// nrs.harvard.edu/urn-3:HUL.InstRepos:dash.current.terms-of-use\#OAP

\section{Share Your Story}

The Harvard community has made this article openly available.

Please share how this access benefits you. Submit a story.

Accessibility 
Draft Version SePtember 17, 2018

Preprint typeset using LATEX style emulateapj v. 08/22/09

\title{
THE IMPRINT OF DISSIPATION ON THE SHAPES OF MERGER REMNANT LOSVDS
}

\author{
Loren Hoffman ${ }^{1}$, Thomas J. Cox ${ }^{2}$, Suvendra Dutta ${ }^{2}$, Lars Hernquist ${ }^{2}$ \\ Draft version September 17, 2018
}

\begin{abstract}
The properties of elliptical galaxies are broadly consistent with simulated remnants of gas-rich mergers between spirals, motivating more detailed studies of the imprint of this formation mechanism on the remnant distribution function. Gas has a strong impact on the non-Gaussian shapes of the line-of-sight velocity distributions (LOSVDs) of the merger remnant, owing to the embedded disk that forms out of the gas that retains its angular momentum during the merger, and the strong central mass concentration from the gas that falls to the center. The deviations from Gaussianity are effectively parametrized by the Gauss-Hermite moments $h_{3}$ and $h_{4}$, which are related to the skewness and kurtosis of the LOSVDs. We quantify the dependence of the $\left(h_{3}, h_{4}\right)-v / \sigma$ relations on the initial gas fraction $f_{\text {gas }}$ of the progenitor disks in 1:1 mergers, using Gadget-2 simulations including star formation, radiative cooling, and feedback from supernovae and AGN. For $f_{\text {gas }} \lesssim 15 \%$ the overall correlation between $h_{3}$ and $v / \sigma$ is weak, consisting of a flat negatively correlated component arising from edge-on viewing angles plus a steep positively correlated part from more face-on projections. The spread in $v / \sigma$ values decreases toward high positive $h_{4}$, and there is a trend toward lower $h_{4}$ values as $f_{\text {gas }}$ increases from 0 to $15 \%$. For $f_{\text {gas }} \gtrsim 20 \%$ the $\left(h_{3}, h_{4}\right)-v / \sigma$ distributions look quite different - there is a tight negative $h_{3}-v / \sigma$ correlation, and a wide spread in $v / \sigma$ values at all $h_{4}$, in much better agreement with observations. Re-mergers of the high- $f_{\text {gas }}$ remnants (representing dry mergers) produce slowly rotating systems with near-Gaussian LOSVDs. We explain all of these trends in terms of the underlying orbit structure of the remnants, as molded by their dissipative formation histories. Subject headings: methods: n-body simulations - galaxies: elliptical and lenticular, cD - galaxies: formation - galaxies: interactions - galaxies: kinematics and dynamics
\end{abstract}

\section{INTRODUCTION}

Many of the observed properties of elliptical galaxies indicate a violent formation history. They are dynamically hot systems, with high velocity dispersions dominating over ordered stellar streaming. Gasrich tidal tails, and rings and shells indicative of the recent disruption of a spiral galaxy, often surround systems otherwise resembling ordinary giant ellipticals (Arp 1966; van Dokkum 2005). These observations first led Toomre \& Toomre (1972) to suggest that elliptical galaxies are the products of mergers between spirals. N-body simulations of mergers between disk galaxies generically reproduce many of the gross properties of observed ellipticals, including density profiles that follow the " $r{ }^{1 / 4}$ " law in projection (van Albada 1982; McGlvnn 1984), flat rotation curves (White 1978; Farouki \& Shapiro 1982), slow rotation and anisotropic velocity distributions (White 1978; Gerhard 1981), and triaxial shapes (Gerhard 1981; Barnes 1988). Today we understand the Toomre merger hypothesis within the broader context of hierarchical structure formation in the Universe - in the favored $\Lambda$ CDM cosmology, an elliptical galaxy-sized halo has typically suffered $\sim 1$ major merger since $z \sim 2-3$, the epoch over which the bulk of its stellar mass formed (e.g. Lacev \& Cole 1993; Manrique \& Salvador-Sole 1996; Kauffmann 1996; De Lucia et al. 2006; Hopkins et al.

\footnotetext{
Electronic address: l-hoffman@northwestern.edu

${ }^{1}$ Department of Physics and Astronomy, Northwestern University, Dearborn Observatory, 2131 Tech Drive, Evanston, IL, 60208

2 Department of Astronomy, Harvard University, 60 Garden Street, Cambridge, MA 02138
}

\section{8a, 2009a; Stewart et al. 2008).}

Observations of elliptical galaxies also show signatures of dissipation in their buildup. The phase space densities in the cores of ellipticals exceed the maximum densities in spirals, implying a violation of $\mathrm{Li}-$ ouville's theorem if ellipticals arise from dissipationless spiral-spiral mergers (Carlberg 1986). This problem is naturally resolved by a gas component that is driven to the center by tidal torques during the merger (Negroponte \& White 1983; Barnes \& Hernquist 1991; Hernquist et al. 1993). Elliptical galaxies also display kinematic features that are most easily explained as signatures of dissipation, such as rapid oblate rotation (e.g. Davies et al. 1983; Bender et al. 1992), embedded disks (Bender 1990; Rix \& White 1990), and kinematically decoupled subsystems (Hernquist \& Barnes 1991; Efstathiou et al. 1982; Franx \& Illingworth 1988; Jedrzeiewski \& Schechter 1988; Krainovic et al. 2008). Cox et al. (2006) showed that a wide variety of the photometric and kinematic properties of observed ellipticals, including their half-light radii and velocity dispersions, flattening, isophotal shapes, velocity anisotropy, and major and minor axis rotation were much better reproduced by an ensemble of simulated $40 \%$ gas disk merger remnants than by the corresponding ensemble of dissipationless mergers. Robertson et al. (2006) found that a gas fraction of $\sim 30 \%$ was needed to match the observed tilt in the "fundamental plane" (Dressler et al. 1987; Diorgovski \& Davis 1987), a value close to the typical gas fraction at $z \sim 2-3$.

The violent relaxation in galaxy mergers is incomplete - substantial memory of the initial conditions is retained. 
For example the remnant may tend to rotate in the sense of the initial orbital angular momentum or disk spins (Barnes 1992; Hernquist 1992, 1993), and an initial stellar metallicity gradient will be blurred but not erased during the merger (White 1978). This incompleteness is enhanced by the presence of gas, which can form cold features in the stellar distribution relatively late in the merger process, which subsequently experience less violent relaxation than features present at the beginning of the merger. This fine structure provides a fossil record of the galaxy's formation history (e.g. Statler 1987).

One way to parametrize the finer features in a galaxy's distribution function, which is well-suited to spectroscopic observations, is through the moments of the lineof-sight velocity distribution (LOSVD) as a function of projected location in the galaxy. For continuous stellar line profiles, these moments are measured by fitting the LOSVD to a Gaussian multiplied by a truncated series of Gauss-Hermite $(\mathrm{GH})$ basis functions $H_{k}(w)$,

$$
F_{G H}\left(v_{l o s}\right) \propto e^{-\frac{1}{2} w^{2}}\left[1+\sum_{k=3}^{n} h_{k} H_{k}(w)\right],
$$

where $w \equiv\left(v_{\text {los }}-\bar{v}\right) / \sigma$ and $\sigma$ is the line-ofsight (LOS) velocity dispersion. This method is less sensitive to noise than computing the moments directly (van der Marel \& Franx 1993; Gerhard 1993; Binney \& Merrifield 1998). For modest deviations from Gaussianity, $h_{3}$ represents the skewness of the distribution (see Binney \& Merrifield 1998, Fig. 11.5) and can, for example, indicate whether the net rotation in a galaxy arises from the dominant orbit population, or a small group of streaming stars. The quantity $h_{4}$ measures the kurtosis; positive $h_{4}$ values generally indicate a radial anisotropy in the velocity distribution, while negative $h_{4}$ indicates a tangential bias (Gerhard 1993). In a nonrotating system, $h_{4}$ can distinguish between a dominant population of radial (box) orbits, and cancelling streams of high-angular momentum (tube) orbits.

In recent years, integral field spectroscopy (IFS) has made it possible to obtain high $\mathrm{S} / \mathrm{N}$, high-resolution $2 \mathrm{D}$ maps of the LOSVD in nearby ellipticals (Bacon et al. 2001; Emsellem et al. 2004). Schwarzschild modelling studies have shown that, in practice, the observed $2 \mathrm{D}$ maps of the first four moments of the velocity distribution, $\left\{v, \sigma, h_{3}, h_{4}\right\}$, are sufficient to uniquely reconstruct the full 3D stellar orbital distribution in most cases (Cappellari et al. 2003; van de Ven et al. 2008; van den Bosch et al. 2008; van den Bosch \& van de Ven 2008). Patterns in the higher moments, $\left\{h_{k}, k \geq 5\right\}$, typically fall below the noise in the observations. It is therefore standard practice to truncate the $\mathrm{GH}$ series at $k=4$, and encapsulate the non-Gaussian shape of the distribution in the two parameters $h_{3}$ and $h_{4}$.

Previous authors (Bendo \& Barnes 2000; Jesseit et al. 2005; Naab et al. 2006; Jesseit et al. 2007) have observed that simulated gas-rich merger remnants occupy different areas of the $h_{3,4}-v / \sigma$ planes from dissipationless diskdisk merger remnants. Dissipationless remnants tend to show an overall positive correlation between $h_{3}$ and $v / \sigma$, while in gas-rich remnants, as in observed ellipticals (Bender et al. 1994; Krainovic et al. 2008), this correlation is negative. Gonzalez-Garcia et al. (2006) show that adding a bulge component to the merging disks can also yield larger $h_{3}$ values and a negative $h_{3}-v / \sigma$ correlation, by allowing the disks to retain more of their initial angular momentum. Bender et al. (1994) demonstrate that the observed $h_{3}-v / \sigma$ relation is too steep to be explained by two-integral oblate rotator models (e.g. Dehnen \& Gerhard 1994), but can easily be accounted for by the superposition of a hot stellar spheroid with a cold embedded disk. This type of distribution function arises naturally from a combination of violent relaxation and dissipation in gas-rich mergers of spiral galaxies (Hopkins et al. 2009b).

Jesseit et al. (2005) and Naab et al. (2006) performed a detailed study of the stellar orbit structure of 1:1 and $3: 1$ merger remnants with 0 and $10 \%$ gas, and its imprint on their photometric and kinematic properties. They found that the gas drives an exchange between box and short-axis tube orbits, making the remnants more oblate in shape. This suppression of box orbits strongly influences the shapes of the LOSVDs, bringing the $h_{3,4}-v / \sigma$ relations into better agreement with observed rapidly rotating ellipticals. Jesseit et al. (2007) showed that 2D kinematic maps of the same set of remnants display many of the intriguing features seen in real galaxies, including misaligned rotation, embedded disks, and kinematically decoupled cores.

In this paper we explore the effect of gas on the $h_{3,4}-v / \sigma$ relations using a version of the tree/SPH code Gadget-2 (Springel 2005) that includes star formation, radiative cooling, and feedback from supernovae and AGN (Springel \& Hernquist 2003; Springel et al. 2005). The inclusion of real-time star formation allows us to consider the higher gas fractions typical of spiral galaxies at the peak elliptical formation epoch, $f_{\text {gas }} \sim 30 \%$ at $z \sim 2$. For fixed $f_{\text {gas }}$, the dissipative signature on the dynamics may be reduced by star formation, if the gas is converted to collisionless material early on in the merger. The dissipational features may also be more spatially extended when star formation is included.

We simulate a representative series of mergers between equal-mass, Milky Way-sized disk galaxies at a series of initial gas fractions ranging from 0 to $40 \%$, as well as re-mergers of the spheroidal remnants. We quantify the dependence of the $h_{3,4}-v / \sigma$ diagrams on $f_{g a s}$, and explain the patterns that we find in terms of the underlying stellar orbital distribution. Our primary goals are: (a) to see whether this diagnostic points to the same typical gas fraction as other indicators, such as the FP tilt and peak quasar redshift; (b) to locate "wet" (gas-rich disk-disk) and "dry" (re-mergers of gas-poor spheroid) mergers in $\left(h_{3,4}-v / \sigma\right)$ space, with the aim of distinguishing these two populations in IFS observations; and (c) to connect the observed trends in the non-Gaussian moments with the underlying orbit structure typical of merger remnants in an intuitive way.

\section{SIMULATIONS AND METHODS}

Our initial galaxy models consist of exponential disks embedded in Hernquist (1990) dark matter halos, with masses comparable to the Milky Way. The galaxy models are described in detail in Springel et al. (2005). The halo concentration and spin parameter were set to $c=0.9$ and $\lambda=0.033$. The disk mass was $4.1 \%$ of the halo mass, and its specific angular momentum was assumed equal to that of the halo. The halo was realized with 120,000 
particles, and 80,000 stars initially comprised the disk. A fraction $f_{\text {gas }}$ of the disk stars were replaced with SPH gas particles at the start of the simulation, and this component could subsequently form new stars if a threshold density $\rho_{\text {th }}$, tuned to match the Schmidt Law (Schmidt 1959; Kennicutt 1998), was exceeded. The gravitational softening length was $140 \mathrm{pc}$, which set a minimum spatial resolution scale in the core. (See also Cox et al. 2006 for further description of the simulations.)

The disks began at a separation of $100 \mathrm{kpc}$, on parabolic orbits with a periapsis distance of $7.1 \mathrm{kpc}$. They typically merged after around 1.5Gyrs, and the simulations were run to a final time $t_{\text {end }}=3 h^{-1}=4.3$ Gyrs. Cox et al. (2006) show that the global remnant properties reach a steady state at one effective radius $\left(R_{e}\right)$ around $0.3 \mathrm{Gyrs}$ after the merger, so by $t_{\text {end }}$ the remnants can safely be considered relaxed at this scale. The set of eight merger trajectories used in this study correspond to orbits $i-p$ in Cox et al. (2006), chosen to uniformly sample the phase space of possible orbits (Barnes 1992).

The series of eight representative disk-disk mergers was performed at seven different gas fractions, $f_{\text {gas }}=0,5$, $10,15,20,30$, and $40 \%$. We also ran two series of remergers of the 20 and $40 \%$ gas remnants, intended to represent dry mergers of realistic gas-poor ellipticals. To produce a representative sample for each re-merger series, we randomly selected eight pairs from among the disk-disk merger remnants, and re-merged them on trajectories $i-p$ again.

For each of the simulated remnants we selected 100 random isotropically sampled viewing angles, and a synthetic 2D map of the kinematics was constructed within $1 R_{e}$ for each LOS. A constant mass-to-light ratio was assumed throughout the analysis. In each spatial bin we constructed a velocity histogram and extracted $v, \sigma$, $h_{3}$, and $h_{4}$ by performing a least-squares fit to the 5 parameter function

$$
\begin{aligned}
F(y)=A \exp \left[-\frac{(y-v)^{2}}{2 \sigma^{2}}\right]\{1 & +h_{3} H_{3}\left(\frac{y-v}{\sigma}\right) \\
& \left.+h_{4} H_{4}\left(\frac{y-v}{\sigma}\right)\right\}
\end{aligned}
$$

using the Levenberg-Marquardt method (Press et al. 1992). We used $40 \times 40$ spatial bins and 80 velocity bins within $\pm 3.5 v_{r m s}$. The Gadget particles were smoothed over a radius $h_{\text {smooth }}=\max \left(h_{\text {see }}, h_{n g b}\right)$, where $h_{\text {see }}=150 p c$ corresponds to a seeing of 1.5 " at $20 \mathrm{Mpc}$, and $h_{n g b}$ is a smoothing length based on the distance to the 128th nearest neighbor. This ensured that the effective particle count in each spatial bin was $\gtrsim 1000$. At $t_{\text {end }}$ we also froze the potential, expanded it in the basis set of Hernquist \& Ostriker (1992), and classified the orbits of the stellar particles in this potential. The results of this exercise are thoroughly presented in (Hoffman et al. 2009).

\section{RESULTS}

\subsection{The $h_{3}-v / \sigma$ distribution}

Our results for the $h_{3}-v / \sigma$ relation are presented in Fig. 1. The shape of the distribution is quite sensitive to $f_{\text {gas }}$ for the disk-disk merger remnants. At low gas fractions the overall correlation between $h_{3}$ and $v / \sigma$ is weak; the distribution has a diamond shape consisting of a flat, negatively correlated component plus a steep, positively correlated one. Although the intrinsic structure changes rapidly from flattened, prolate-triaxial systems dominated by box orbits to rounder, more oblate systems dominated by short axis $(z-)$ tube orbits as the gas fraction increases from 0 to $15 \%$ (Hoffman et al. 2009), there is no strong signature of this transformation in the asymmetric $h_{3}$ moment.

For $f_{\text {gas }} \gtrsim 20 \%$ the shape of the $h_{3}-v / \sigma$ distribution changes rather abruptly. These gas-rich remnants are characterized by high $h_{3}$ moments that are strongly anti-correlated with $v / \sigma$, in better agreement with observed fast-rotator ellipticals (Bender et al. 1994; Krajnovic et al. 2008). The observations thus favor typical progenitor gas fractions $\gtrsim 25 \%$, in agreement with other indicators such as the shapes of the remnants (Cox et al. 2006; Hoffman et al. 2009), the twocomponent structure of ongoing mergers (Hopkins et al. 2008b), cuspy (Hopkins et al. 2009c) and cored ellipticals (Hopkins et al. 2009d), and the tilt of the FP (Robertson et al. 2006; Hopkins et al. 2008c). In the SAURON data there is also evidence for a second population of fast rotators with low $h_{3}$ values (Krajnovic et al. 2008); these systems cannot be explained by the simulations performed in this study.

Re-mergers of the high-gas fraction remnants still show a distinct negative correlation between $h_{3}$ and $v / \sigma$, but the remnant LOSVDs are much more concentrated near the origin of the $h_{3}-v / \sigma$ plane. Note the much lower spread in re-merger $h_{3}$ values at $v=0$ compared to diskdisk mergers of any gas fraction. This result is not surprising, since violent relaxation in the re-merger tends to blur out structure in the distribution function and drive the LOSVDs closer to a thermal distribution. Fig. 1 quantifies the degree of thermalization in a single dry merger.

In Fig. 2 we illustrate how the positive and negatively correlated components arise in the low- $f_{\text {gas }}$ remnants, and how the strong $h_{3}-v / \sigma$ anti-correlation arises in the gas-rich ones. In dissipationless remnant $l$ (top six panels), the major-axis $(x-)$ tube orbits produce substantial minor axis rotation even though they only comprise $\sim 20 \%$ of the stellar mass within $1 R_{e}$, since they are highly streaming. In a pixel near the minor axis in an edge-on projection (looking along the intermediate axis), $x$-tubes dominate the luminosity, and when combined with the $z$-tube and box orbits peaked at $v=0$ they result in a distribution with an extended tail in the direction opposite the mean velocity. Since the velocity distribution of the $x$-tube orbits is not too strongly peaked, the $h_{3}$ value is not very high and the $h_{3}-v / \sigma$ relation is relatively flat.

When the same remnant is viewed face-on (projected along the short axis), the three orbit classes are all piled on top of each other, and the streaming $x$-tubes form a bump in the tail of the dominant distribution of boxes and $z$-tubes. We thus get an extended tail in the same direction as the mean velocity, i.e. a positive $h_{3}-v / \sigma$ correlation. This high- $v$ bump in the tail produces a stronger asymmetry in the LOSVD while the velocities in the face-on projection tend to be lower, so this effect produces a much steeper $h_{3}-v / \sigma$ relation than the effect illustrated in the top row.

The locus of face-on and edge-on projections in the 


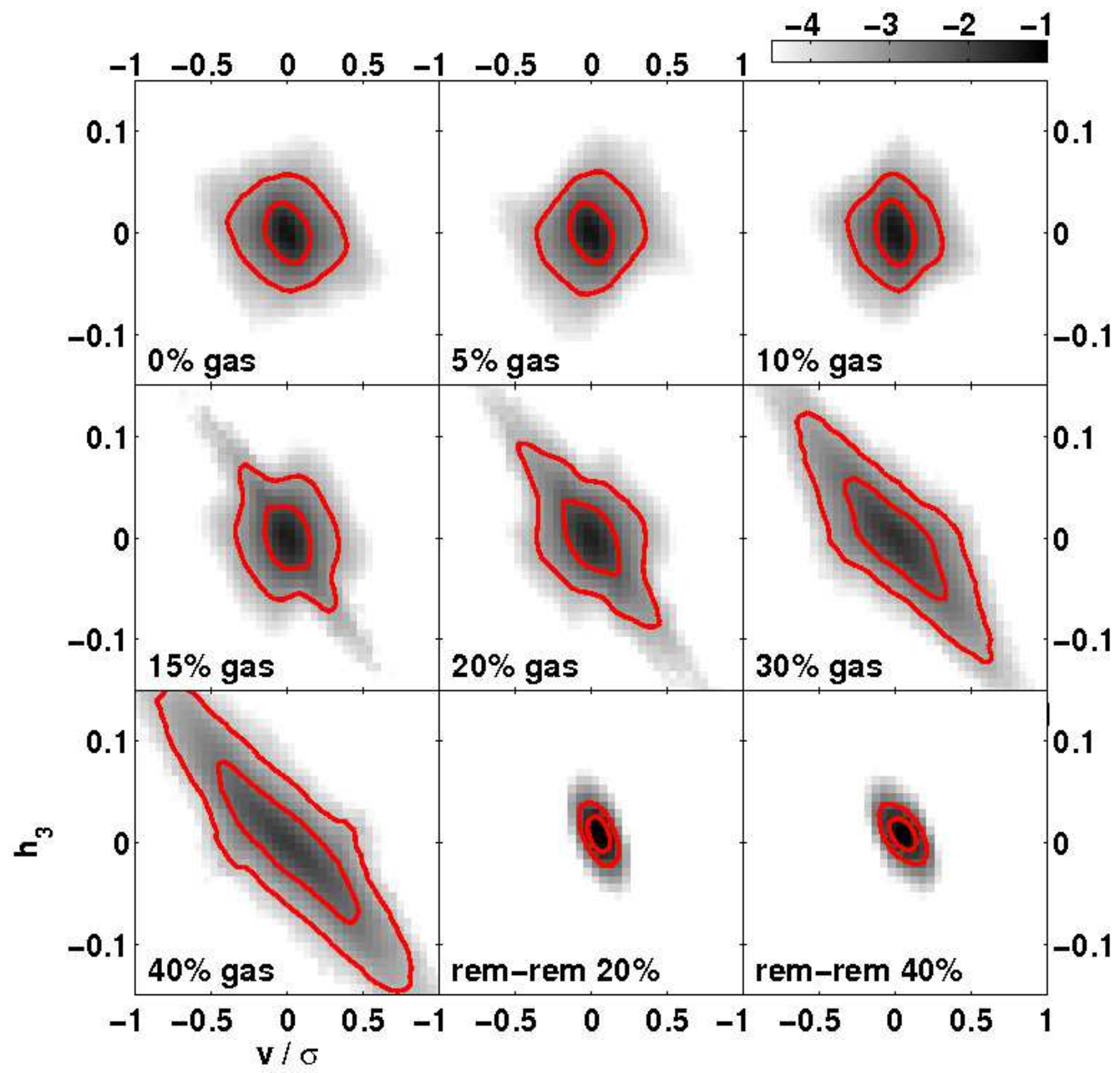

FIG. 1.- Local $h_{3}-v / \sigma$ relation for each of our nine merger simulation sets. The first seven panels are for disk-disk mergers of different gas fractions, while the last two panels (lower right) are for dry mergers of the 20 and $40 \%$ gas remnants. Each map is averaged over the eight unbiased merger orbits, and 100 isotropically selected lines of sight per remnant. The shading is proportional to the logarithm of the luminosity in each $h_{3}-v / \sigma$ bin, normalized so that the entire map sums to one. The red lines are contours containing 68 and $95 \%$ of the luminosity.

$h_{3}-v / \sigma$ diagram, averaged over all remnants of a given gas fraction, is shown in the bottom row of Fig. 2. To illustrate the trends we show one set of low- $f_{\text {gas }}$ disk-disk mergers, one set of high- $f_{\text {gas }}$ disk-disk mergers, and one set of re-merger remnants. As indicated in the above example, face-on projections dominate a steep positivelycorrelated component, while edge-on projections dominate the shallower negatively correlated component. The positively correlated component is relatively suppressed at high gas fractions.

In gas-rich remnants the dominant population (z-tube orbits) is streaming as in the edge-on dissipationless rem- nant, only now the streaming is much stronger. This effect arises from the embedded disk that re-forms after the merger out of the gas that did not lose its angular momentum and fall to the center (Hopkins et al. 2009b). The red and blue dashed lines in the bottom LOSVD show the contribution from "old" stars put in as collisionless particles at the beginning of the simulation, and from the $15 \%$ of the new stars (formed out of gas during the merger) that formed latest. These late-forming stars comprise only a few percent of the total stellar mass, but are prominent in spatial bins lying along the major axis. These disk stars have a much lower velocity dispersion 

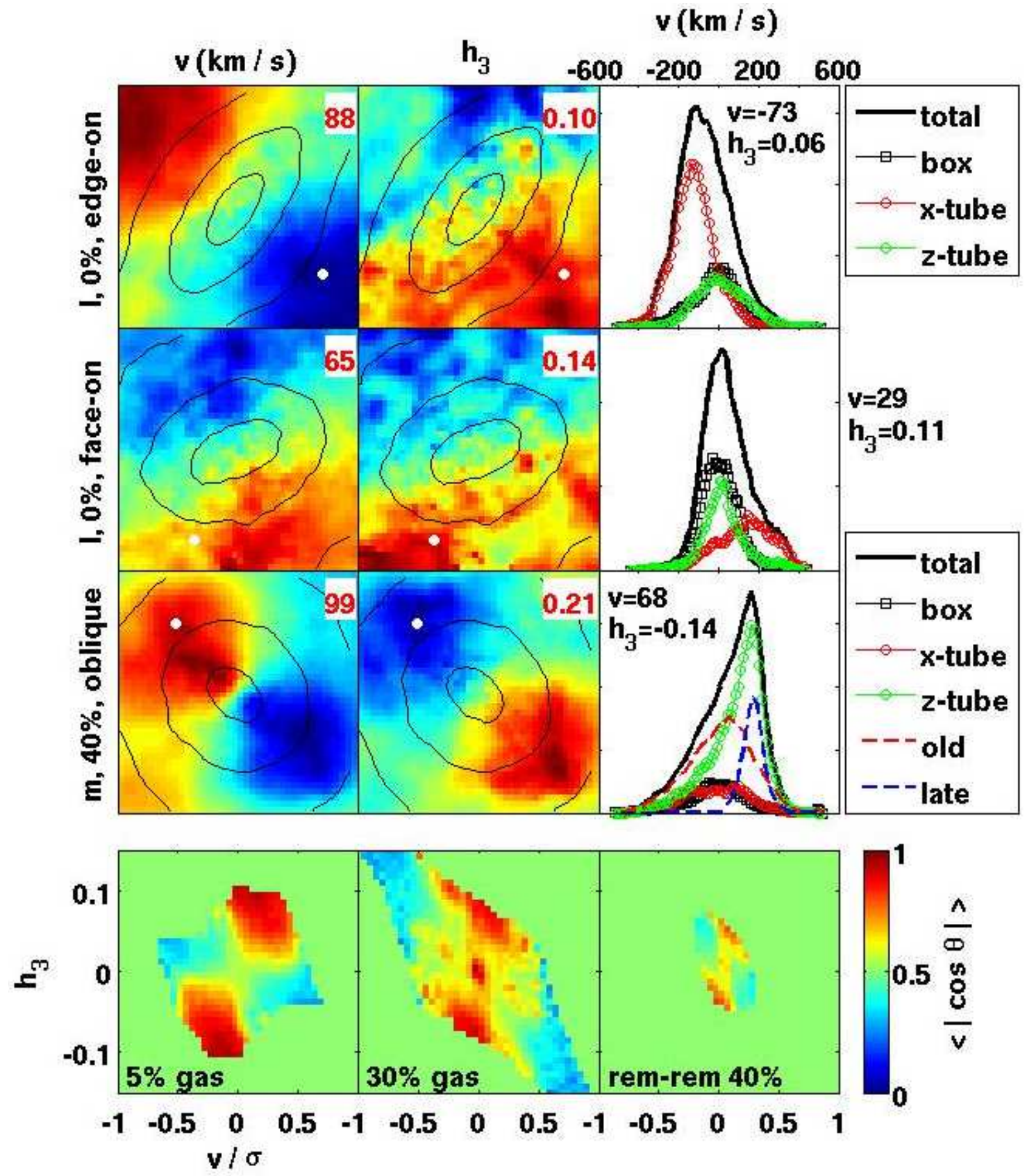

Fig. 2.- Explaining the $h_{3}-v / \sigma$ trends. Top row: Merger orbit $l, 0 \%$ gas, at a nearly edge-on viewing angle. Second row: Same remnant, viewed nearly face-on. Third row: Merger orbit $m, 40 \%$ gas, viewed at an angle about $30^{\circ}$ from edge-on. First two columns: $v$ and $h_{3}$ maps within $1 R_{e}$. The color scale runs from $-h_{\max }$ to $h_{\max }$, where $h_{\max }$ is the maximum value of the moment in any pixel, and the number in the upper right corner gives the value of $h_{\max }$. The black contours are over-plotted isophotes. The third column shows the smoothed LOSVD at the location specified by the white dot on the $v$ and $h_{3}$ maps. The heavy black line is the full LOSVD, while the red and green circles and black squares break it down by orbit class. In the case of the gas-rich remnant (bottom row), we also show the separate contributions from old stars (red dashed line) and the $15 \%$ of the new stars that formed latest (blue dashed line). (See the text for further discussion of these plots.) Bottom row: Net contribution of face-on and edge-on projections to the $h_{3}-v / \sigma$ distributions. The three maps correspond to the second, sixth, and ninth panels of Fig. 1, but now each bin is colored according to the luminosity-weighted mean $|\cos \theta|$, where $\theta$ is the polar angle between the LOS and the intrinsic short axis of the triaxial remnant. $|\cos \theta|=1$ means perfectly face-on, while $|\cos \theta|=0$ is perfectly edge-on. 
than the remnant as a whole, and produce a sharp high- $v$ peak in the dominant $z$-tube population, giving a distribution that is strongly skewed in the sense opposite the mean velocity.

As a rule, disk-disk mergers tend to produce large asymmetric deviations from Gaussianity owing to subcomponents that retain memory of the initial streaming of the disk stars, and to gas that is redistributed and spun up through dissipation to form a thin embedded disk. The $h_{3}-v / \sigma$ correlation is generally negative when the dominant orbit population tends to stream, and positive when the rotation is caused by sub-dominant streaming population superimposed on the tail of the dominant orbits.

\subsection{The $h_{4}-v / \sigma$ relation}

We now turn to the symmetric deviations of the LOSVDs from a Gaussian shape, measured by the "kurtosis" parameter $h_{4}$. In Fig. 3 we present the $h_{4}-v / \sigma$ relations from our simulations. For $f_{\text {gas }} \lesssim 15 \%$ the $h_{4}-v / \sigma$ distribution has a triangular shape that narrows sharply toward high positive $h_{4}$. Note that this is the naive expectation for a single population of tube orbits - viewed edge on the high-angular momentum orbits tend to pile up in the tails of the velocity distribution and any significant imbalance in the sense of their rotation produces a high $v / \sigma$, while viewed faceon they pile up in a peak around $v=0$. However this pattern differs sharply from IFS observations, in which a mild increase in the $v / \sigma$ spread is actually seen toward high $h_{4}$ (Krajnovic et al. 2008). There is also a trend toward lower $h_{4}$ values as $f_{\text {gas }}$ increases from 0 to $15 \%$, arising from the conversion of box orbits into $z$-tubes by the central mass concentration formed by the gas that falls to the center and produces the starburst (Barnes \& Hernquist 1996; Mihos \& Hernquist 1994; Naab et al. 2006).

We get a marked change in the shape of the $h_{4}-v / \sigma$ distribution at $f_{\text {gas }} \sim 20 \%$, as we did for the $h_{3}-v / \sigma$ relation. At higher gas fractions we get strong positive as well as moderate negative $h_{4}$ values over a wide range in $v / \sigma$ - the $v / \sigma$ spread actually increases mildly toward high $h_{4}$ as in observed systems.

Like the transformation in the $h_{3}-v / \sigma$ distribution, this rapid transition owes to the appearance of a strong disk component starting at $f_{\text {gas }} \sim 20 \%$, as shown in Fig. 4. In high- $v / \sigma$, edge-on projections of the disk, $h_{4}$ is influenced by two competing effects - the strong negative kurtosis of the more isotropic population of old stars on $z$-tube orbits, and the strongly peaked population of late-forming disk stars that tend to produce a positive $h_{4}$ in superposition with the old component. In the pixel shown in the top row of Fig. 4, the latter effect wins out. Although the old stellar $z$-tube population has a negative kurtosis, the narrow superimposed peak from the late-formed disk gives the full LOSVD a net positive $h_{4}$. This positive $h_{4}$ is greatly enhanced for the $z$-tube orbits alone. In the outer dotted pixel whose LOSVD is shown in the middle row, the disk component is somewhat weaker and the negative kurtosis of the old $z$-tube population wins, giving the full distribution a net negative $h_{4}$.

This example demonstrates how we may get a wide spread in $h_{4}$, including strong positive as well as negative values, at high $v / \sigma$. We caution the reader that in this situation $h_{4}$ is quite sensitive to small changes in the disk strength, and the GH fitting is sensitive to noise, so care must be taken in quantitative comparisons between simulations and observations.

In the $h_{4}-v / \sigma$ diagram the re-mergers are once again more concentrated around the origin, for the same reason discussed in the previous subsection. The smaller nonGaussian moments of the re-mergers at fixed $v / \sigma$ suggest one way to separate re-merger remnants from other slow rotators. The re-mergers tend to have LOSVDs with positive $h_{4}$, also an expected signature of violent relaxation, which generally results in a radially-biased distribution function (Binney \& Tremaine 2008).

Face-on projections generically give high positive $h_{4}$ values, as both the box and $z$-tube orbits are strongly peaked around $v=0$ in this projection. Particularly strong positive $h_{4}$ values can arise in face-on views of gasrich remnants since the latest-formed stars form a narrow peak centered on $v=0$, as illustrated in the third row of Fig. 4. The bottom row of this figure shows the average locus of edge-on and face-projections in the $v / \sigma$ plane face-on projections dominate the high- $h_{4}$, low $v / \sigma$ part of the diagram, while edge-on projections dominate the high $v / \sigma$ and low $h_{4}$ regions, as well as the high $v / \sigma$, high $h_{4}$ wings in the gas-rich remnants.

\section{DISCUSSION AND CONCLUSIONS}

Mergers between spiral galaxies generically produce remnants with significantly non-Gaussian LOSVDs, owing to the presence of subcomponents that retain memory of the initial conditions. This effect is especially pronounced in mergers with $f_{\text {gas }} \gtrsim 20 \%$ where the dissipative component re-forms an embedded disk, producing a strong negative $h_{3}-v / \sigma$ correlation and a wide spread of $h_{4}$ values at high $v / \sigma$. Both of these features are in agreement with observations of elliptical galaxies (Bender et al. 1994; Krainovic et al. 2008).

Upon closer examination the SAURON fast-rotator population appears to consist of two subgroups, one with a steep $h_{3}-v / \sigma$ correlation, and a second with low $h_{3}$ for all $v / \sigma$ (Krajnovic et al. 2008). A gas-rich major merger scenario cannot account for the second group, which suggests that the two fast-rotator populations may differ in their formation mechanisms.

Dry mergers produce slowly rotating remnants with small $h_{3}$ and $h_{4}$, as violent relaxation blurs features in the distribution function and partially thermalizes the local velocity distribution. This provides one way to distinguish dry merger remnants within the population of slowly rotating ellipticals. The large spread in $h_{3}$ and $h_{4}$ among the SAURON slow rotators (Krajnovic et al. 2008) suggests that the slow rotators are not all dry merger remnants, as has been proposed to explain the fast-slow rotator dichotomy Gonzalez-Garcia et al. 2006; Kormendy et al. 2008).

At fixed redshift a tight correlation is observed between the gas fraction and stellar mass of late-type galaxies, with $f_{\text {gas }}$ varying from $\sim 50 \%$ at $\mathrm{M}_{\text {star }}=10^{10} \mathrm{M}_{\odot}$ to $\sim 10 \%$ at $\mathrm{M}_{\text {star }}=3 \times 10^{11} \mathrm{M}_{\odot}$ at $z \sim 2$ (Stewart et al. 2009 and references therein). The $f_{\text {gas }}$ labels in Figs. 1 and 3 may therefore be taken loosely as a proxy for galaxy mass, and our gas-rich merger model predicts a variation in the $h_{3,4}-v / \sigma$ relations with galaxy luminosity. 


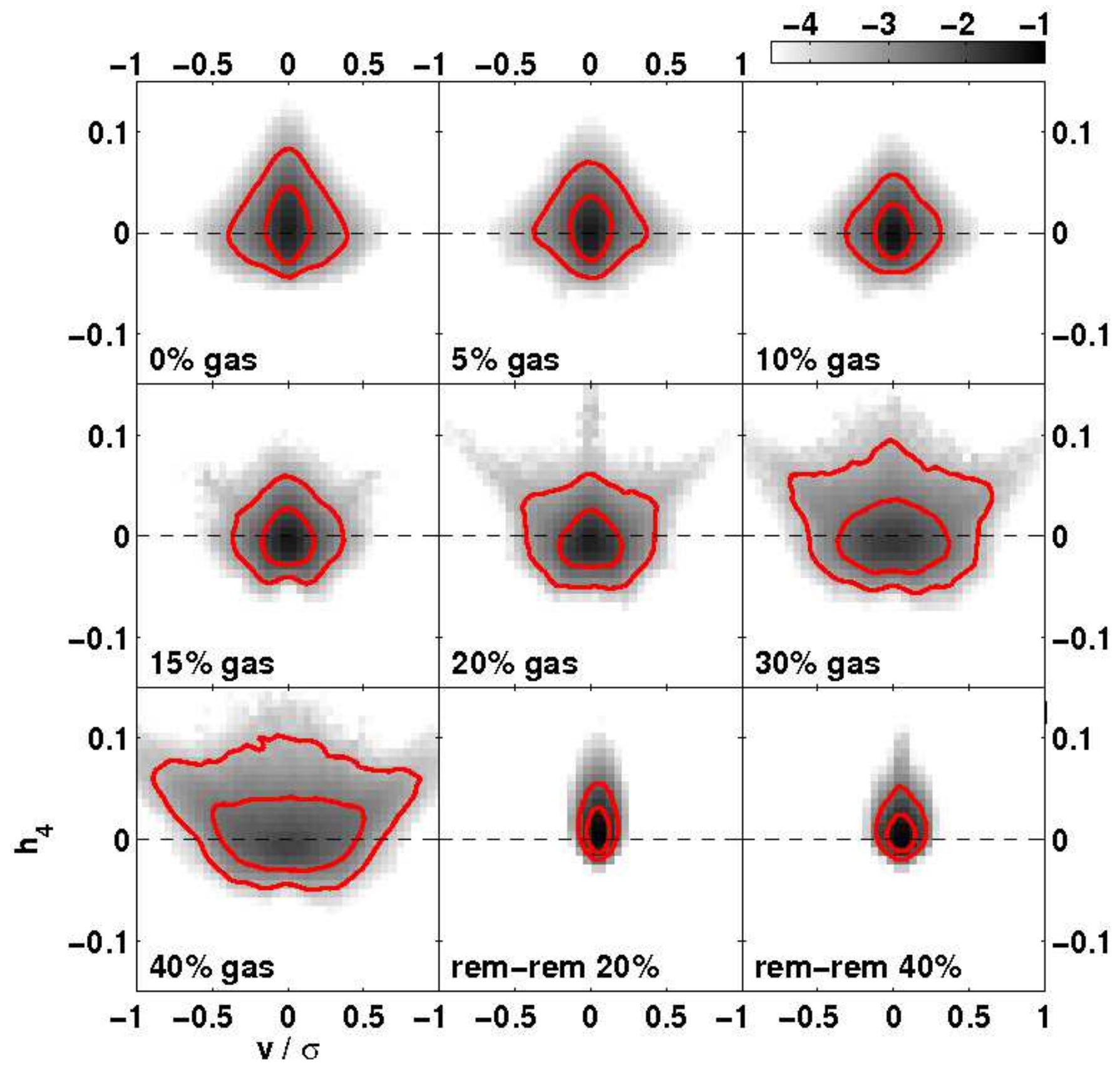

FIG. 3.- Local $h_{4}-v / \sigma$ relation for each of our simulation sets. The shading and contours are as in Fig. 1.

In this paper we considered only a very restricted set of merger models, namely binary 1:1 mergers of bulgeless disks. The embedded disk component in our remnants may be artificially pronounced because real galaxies would suffer subsequent harassment through minor mergers, interactions with satellites, and secular processes. Some of the effects of increasing $f_{\text {gas }}$ (e.g. higher $v / \sigma$ and $\left.h_{3}\right)$ can also be achieved by decreasing the merger mass ratio (Bournaud et al. 2005; Gonzalez-Garcia et al. 2006; Naab et al. 2006; J Jesseit et al. 2007, 2009), and it is important to disentangle these trends. Extensions of this work include varying the mass ratios and bulge fractions, simulating cosmologically-motivated merger se- quences (Burkert et al. 2008; Valluri et al. 2009), and including secular heating processes.

Ultimately we hope to make rigorous statistical comparisons between large libraries of simulations and IFS data. This will require a careful consideration of observational selection biases as well as systematics associated with the GH fitting procedure in the presence of observational noise as well as particle discreteness in the simulations. This study motivates such work by illustrating how detailed 2D kinematic information can reveal the underlying orbit structure of elliptical galaxies, and place stronger constraints on models of their formation. 

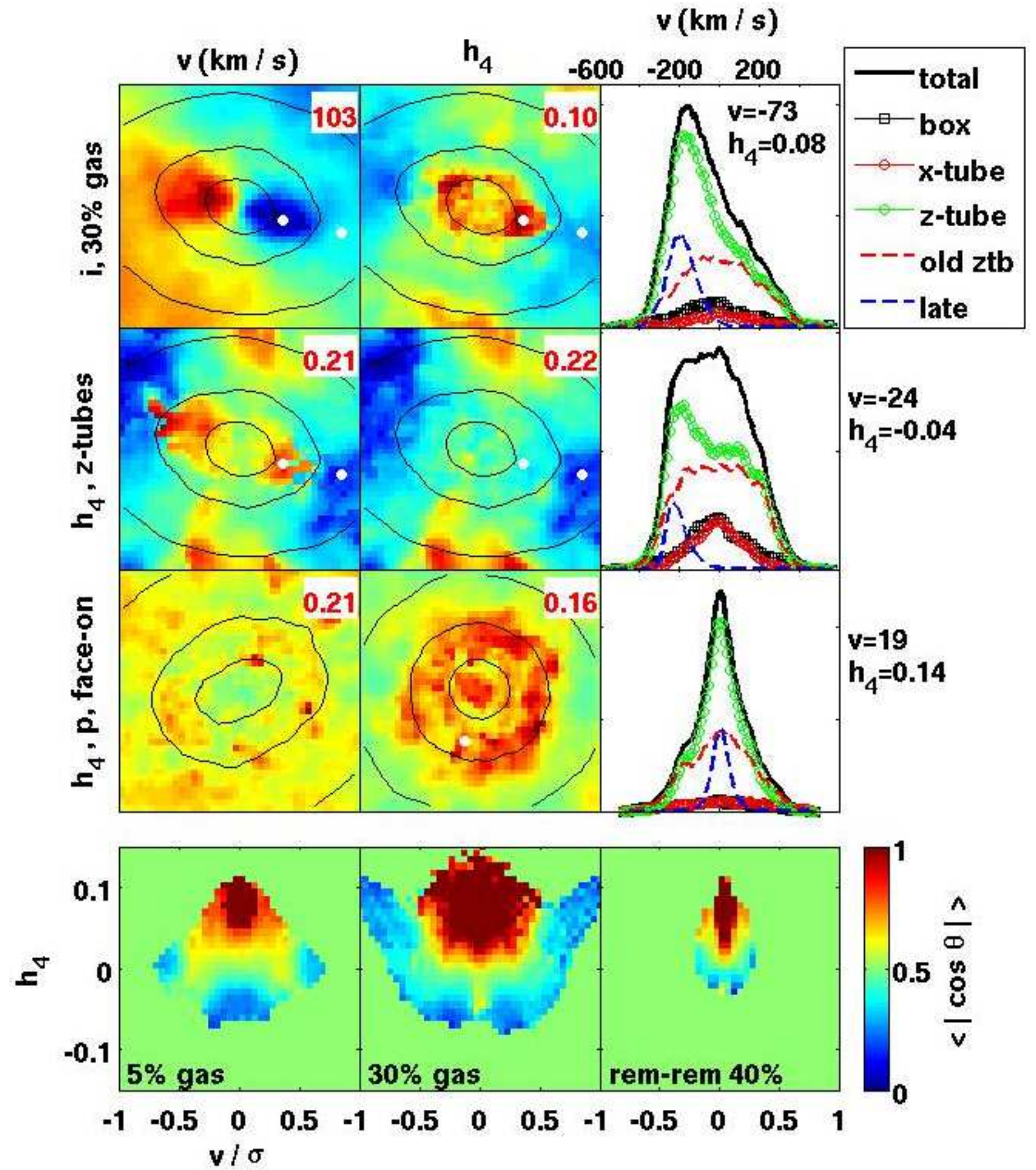

Fig. 4. - Explaining the $h_{4}-v / \sigma$ relation. The top six panels are from merger orbit $i$, viewed about $15^{\circ}$ from edge-on. First row: $v$ and $h_{4}$ maps for this remnant. Second row: $h_{4}$ maps for the $z$-tube orbits alone. The left map is for all of the stars on $z$-tube orbits, while the right map includes only old stars on $z$-tube orbits, excluding those that formed from gas during the merger. In the right-hand column the LOSVDs are shown at the two points indicated by the white dots, broken down by orbit class and stellar age as in Fig. 2 . Third row: $h_{4}$ maps for merger orbit $p$, viewed face-on, at $0 \%$ gas (left) and $40 \%$ gas (right). The LOSVD at the dotted location on the $40 \%$ gas map is shown in the third column. Bottom row: Net contribution of face-on and edge-on projections to the $h_{4}-v / \sigma$ distribution, plotted as in the bottom row of Fig. 2. 
We would like to thank Bart Willems for technical help, and Glenn van de Ven for useful discussions. This work was supported in part by a Lindheimer Postdoctoral Fellowship at Northwestern University. Computations were performed on the Fugu computer cluster funded by NSF
MRI grant PHY-0619274 to Vicky Kalogera, and the Sauron computer cluster at the parallel computing center of the Institute for Theory and Computation at the Harvard-Smithsonian Center for Astrophysics.

\section{REFERENCES}

Arp, H. 1966, Atlas of Peculiar Galaxies, (Pasadena: California Institute of Technology)

Bacon, R. et al. 2001, MNRAS, 326, 23

Barnes, J.E. 1988, ApJ, 699, 717

Barnes, J.E. 1992, ApJ, 393, 484

Barnes, J.E. \& Hernquist, L.E. 1991, ApJL, 370, 65

Barnes, J.E. \& Hernquist, L.E. 1996, ApJ, 471, 115

Bendo, G.J. \& Barnes, J.E. 2000, MNRAS, 316, 315

Bender, R. 1990, A\&A, 229, 441

Bender, R., Burstein, D., \& Faber, S.M. 1992, ApJ, 399, 462

Bender, R., Saglia, R.P., \& Gerhard, O.E. 1994, MNRAS, 269, 785

Binney, J. \& Merrifield, M. 1998, Galactic Astronomy, (Princeton: Princeton University Press)

Binney, J. \& Tremaine, S. 2008, Galactic Dynamics, (Princeton: Princeton University Press)

Bournaud, F., Jog, C.J., \& Combes, F. 2005, A\&A, 437, 69

Burkert, A., Naab, T., Johansson, P.H., \& Jesseit, R. 2008, ApJ, 685,897

Cappellari, M. et al. 2003, in Carnegie Observatories Astrophysics Series, Vol. 1: Coevolution of Black Holes and Galaxies, ed. L. C. Ho (Pasadena: Carnegie Observatories), [arXiv: 0302274]

Carlberg, R.G. 1986, ApJ, 310, 593

Cox, T.J., Dutta, S.N., Di Matteo, T., Hernquist, L., Hopkins, P.F., Robertson, B., \& Springel, V. 2006, ApJ, 650, 791

Davies, R. L., Efstathiou, G., Fall, S. M., Illingworth, G., \& Schechter, P. L. 1983, ApJ, 266, 41

De Lucia, G., Springel, V., White, S.D.M., Croton, D., \& Kauffmann, G. 2006, MNRAS, 366, 499

Dehnen, W. \& Gerhard, O.E. 1994, MNRAS, 268, 1019

Djorgovski, S. \& Davis, M. 1987, ApJ, 313, 59

Dressler, A., Lynden-Bell, D., Burstein, D., Davies, R. L., Faber, S. M., Terlevich, R., \& Wegner, G. 1987, ApJ, 313, 42

Efstathiou, G., Ellis, R. S., \& Carter, D. 1982, MNRAS, 201, 975

Emsellem, E. et al. 2004, MNRAS, 352, 721

Farouki, R.T. \& Shapiro, S.L. 1982, ApJ, 259, 103

Franx, M. \& Illingworth, G.D. 1988, ApJL, 327, 55

Gerhard O.E. 1981, MNRAS, 197, 179

Gerhard, O.E. 1993, MNRAS, 265, 213

Gonzalez-Garcia, A.C., Balcells, M., \& Olshevsky, V.S. 2006, MNRAS, 372, 78

Hernquist, L.E 1990, ApJ, 356, 359

Hernquist, L.E 1992, ApJ, 400, 460

Hernquist, L.E 1993, ApJ, 409, 548

Hernquist, L.E. \& Barnes, J.E. 1991, Nature, 354, 210

Hernquist, L.E \& Ostriker, J.P. 1992, ApJ, 386, 375

Hernquist, L.E, Spergel, D.N \& Heyl, J.S. 1993, ApJ, 416, 415

Hoffman, L., Hernquist, L.E., \& Cox, T.J. 2009, ApJ, in preparation

Hopkins, P.F., Cox, T.J., Keres, D. \& Hernquist, L. 2008a, ApJS, 175,390

Hopkins, P.F., Hernquist, L., Cox, T.J., Dutta, S.N. \& Rothberg, B. 2008b, ApJ, 679, 156

Hopkins, P.F., Cox, T.J. \& Hernquist, L. 2008c, ApJ, 689, 17
Hopkins, P.F., et al. 2009a, MNRAS, 397, 802

Hopkins, P.F., Cox, T.J., Younger, J., \& Hernquist, L. 2009b, ApJ, 691, 1168

Hopkins, P.F., Cox, T.J., Dutta, S.N., Hernquist, L., Kormendy, J. \& Lauer, T.R. 2009c, ApJS, 181, 135

Hopkins, P.F., Lauer, T.R., Cox, T.J., Hernquist, L. \& Kormendy, J. 2009d, ApJS, 181, 486

Jedrzejewski, R. I. \& Schechter, P. L. 1988, ApJL, 330, 87

Jesseit, R., Naab, T., \& Burkert, A. 2005, MNRAS, 360, 1185

Jesseit, R., Naab, T., Peletier, R.F., \& Burkert, A. 2007, MNRAS, 376, 997

Jesseit, R., Cappellari, M., Naab, T., Emsellem, E., \& Burkert, A. 2009, MNRAS, 397, 1202

Kauffmann, G. 1996, MNRAS, 281, 487

Kennicutt, R.C. 1998, ApJ, 498, 541

Kormendy, J., Fisher, D.B., Cornell, M.E., \& Bender, R. 2008, ApJS, 182, 216

Krajnovic, D., et al. 2008, MNRAS, 388, 1537

Lacey, C. \& Cole, S. 1993, MNRAS, 262, 627

Manrique, A. \& Salvador-Sole, E. 1996, ApJ, 467, 504

McGlynn, T.A. 1984, ApJ, 281, 13

Mihos, J.C. \& Hernquist, L. 1994, ApJL, 437, 47

Naab, T., Jesseit, R., \& Burkert, A. 2006, MNRAS, 372, 839

Negroponte, J. \& White, S.D.M. 1983, MNRAS, 205, 1009

Press, W.H., Flannery, B.P., Teukolsky, S.A., Vetterling, W.T. 1998, Numerical Recipes in C: The Art of Scientific Computing, (New York: Cambridge University Press)

Rix, H.-W. \& White, S.D.M. 1990, ApJ, 362, 52

Robertson, B., Cox, T.J., Hernquist, L., Franx, M., Hopkins, P.F., Martini, P., \& Springel, V. 2006, ApJ, 641, 21

Schmidt, M. 1959, ApJ, 129, 243

Springel, V. 2005, MNRAS, 364, 1105

Springel, V., Di Matteo, T., \& Hernquist, L. 2005, MNRAS, 361, 776

Springel, V. \& Hernquist, L., MNRAS 2003, 339, 289

Statler, T.S. 1987, ApJ, 321, 113

Stewart, K.L., Bullock, J.S., Wechsler, R.H., \& Maller, A.H. 2009, ApJ, 702, 307

Stewart, K.L., Bullock, J.S., Wechsler, R.H., Maller, A.H., \&

Zentner, A.R. 2008, ApJ, 683, 597

Toomre, A. \& Toomre, J. 1972, ApJ, 178, 623

Valluri, M., Debattista, V.P., Quinn, T., \& Moore, B. 2009,

MNRAS, submitted, arXiv:0906.4784

van Albada, T.S. 1982, MNRAS, 201, 939

van de Ven, G., de Zeeuw, P.T., \& van den Bosch, R.C.E. 2008, MNRAS, 385, 614

van den Bosch, R.C.E. \& van de Ven, G. 2008, MNRAS, 398, 1117 van den Bosch, R.C.E., van de Ven, G., Verolme, E.K.,

Cappellari, M., \& de Zeeuw, P.T. 2008, MNRAS, 2008, 385

van der Marel, R.P. \& Franx, M. 1993, ApJ, 407, 525

van Dokkum, P.G. 2005, AJ, 130, 2647

White 1978, MNRAS, 184, 185 